\title{
Occlusal status of implant superstructures at mandibular first molar immediately after setting
}

\author{
Yukihiko Okada*, Yuji Sato, Noboru Kitagawa, Keiichiro Uchida, Tokiko Osawa, Yoshiki Imamura and \\ Mayumi Terazawa
}

\begin{abstract}
Background: Occlusal contact on the implant superstructures is important for successful treatment. The purpose of this study was to investigate the occlusal contact of single implant superstructures at the mandibular first molar immediately after seating from weak to strong clenching.

Methods: Subjects were nine patients who had just been fitted with an implant prosthesis in the mandibular first molar region, with no missing teeth other than in the implant region. First, while masseter muscle activity was monitored, maximum clenching strength (100\% maximum voluntary contraction (MVC)) was determined with an electromyogram. Next, occlusal load and occlusal contact area were measured three times at clenching intensities of 40,60, 80, and $100 \%$ MVC by the use of pressure-sensitive film for occlusal force diagnostic and Occluzer for occlusal force measurement. Finally, the occlusal contact area was measured once each at 20,40, and $60 \%$ MVC using a silicone testing material and BiteEye for occlusal contact measurement. A two-way analysis of variance (ANOVA) was used to determine occlusal loading and occlusal area as dependent variables, and clenching strength and presence or absence of implant as between-subject factors. A multiple comparison test was performed using the Bonferroni method.
\end{abstract}

Results: The occlusal contact area and occlusal load of the implant prosthesis increased with clenching strength, and the increases in occlusal contact area and occlusal load of the implant prosthesis were less than those of the contralateral tooth at high clenching strength. However, significant difference was not observed when compared with both sides of the molar region regardless of clenching strength.

Conclusions: The occlusal contact area of the implant had a tendency to be adjusted smaller than the natural tooth by a dental technician and a dentist. On the other hand, despite the small tissue displaceability of the implant, occlusal load on the implant prosthesis was smaller than on the natural tooth at high clenching strength.

Keywords: Occlusal load; Occlusal contact area; Implant; Clenching strength; Occluzer; BiteEye

\section{Background}

Implants maintain osseointegration with the inside of the bone over long periods of time, and mechanical factors are important for retaining function [1]. Particularly, when natural teeth and implants are present together, differences in tissue displaceability when they are subjected to occlusal force may cause dynamic imbalance from strong to weak clenching [2-4].

Therefore, there is a school of thought that prosthetic implants should be given a lower occlusion than natural

\footnotetext{
* Correspondence: okada.yuki@dent.showa-u.ac.jp

Department of Geriatric Dentistry, School of Dentistry Showa University, 2-1-1 Kitasenzoku, Ota Ward, Tokyo 145-8515, Japan
}

teeth [5-7]. On the other hand, from the perspective of jaw function, it is also argued that prosthetic implants should be given the same occlusion as intact natural teeth neighboring the implant [8]. In a study using twodimensional finite element analysis, Maezawa et al. [9] suggested that, even if the occlusal surface of the prosthetic implant is made lower than the occlusal plane, high clenching intensity can result in an excessive occlusal load on the prosthetic implant. In addition, Koyama et al. [10] found no significant differences in occlusal contact point between prosthetic implants and natural teeth, even if clenching intensity varies. From these studies, it appears that the occlusal contact of implant

\section{穴}


prostheses is the same level on natural teeth, regardless of clenching strength, and that occlusal load on implant prosthesis increases more than contralateral natural teeth as clenching strength increases. Methods of quantitative evaluation used to clarify mechanical factors of implant prostheses include pressure-sensitive films for occlusal force diagnostic use and silicone testing materials, but few studies have examined the occlusal contact from weak to strong clenching strength under the same conditions.

In this department to date, Okuyama et al. [11] used a pressure-sensitive film to examine the occlusal contact and to calculate the mean occlusal gap and occlusal load of implants that had progressed satisfactorily, as well as natural teeth. Imamura et al. [12] used a new silicone test material and a pressure-sensitive film to develop a method for investigating changes in occlusal contact from weak to strong clenching intensity in subjects with natural dentition.

Occlusal contact is a reflection of the degree of functional recovery and measurement of factors such as number of occlusal contact points, contact area, and distribution. Occlusal center is advisable before and after treatment [13]. Therefore, the purpose of this study was to investigate the occlusal contact of single implant superstructures at the mandibular first molar immediately after seating by using two different materials with an electromyogram. Ultimately, we aimed to obtain clinical suggestions for the occlusion that should be given to prosthetic implants through a longitudinal follow-up study.

\section{Methods}

\section{Subjects and dentition}

Subjects were nine outpatients (5 male, 4 female) attending Showa University Dental Hospital, aged 35-69 years (mean age, $49 \pm 12$ years) (Table 1). All subjects were cases of single implants placed at the mandibular first molar deficit, on the day of implant prosthesis setting and occlusal adjustment. The criteria for subject

Table 1 Site of implants

\begin{tabular}{lllll}
\hline No. & Sex & Age & Implant system & Implant site \\
\hline 1 & F & 35 & Straumann & Right \\
2 & F & 39 & Brånemark & Left \\
3 & F & 47 & Brånemark & Left \\
4 & F & 49 & Brånemark & Left \\
5 & M & 43 & Straumann & Right \\
6 & M & 46 & Straumann & Right \\
7 & M & 69 & Straumann & Right \\
8 & M & 48 & Brånemark & Left \\
9 & M & 67 & Brånemark & Right \\
\hline
\end{tabular}

selection were no inflammatory symptoms (redness, swelling) in the implantation area [14, 15], no implant mobility, no subjective symptoms, and no noticeable resorption on X-ray photographs.

The requirements for dentition were that other than the implant, which was the tooth to be studied, all teeth were natural; 28 teeth were present from the central incisors to the second molars, which includes the implant area; there were no mobile teeth; a basic periodontal test showed no pockets of $4 \mathrm{~mm}$ or more; there was no history of orthodontic therapy; and there was no oromandibular dysfunction such as temporomandibular disorder, masticatory muscle pain, or mandibular movement abnormality. In addition, occlusal adjustment was performed by a doctor in attendance. The implant prosthesis had one or more occlusal contact points at maximum clenching strength. Moreover, it has been adjusted as not to interfere with existing guide during lateral movement. Fourteen patients were selected based on these conditions, and consent was obtained from the doctor in charge of the 12 patients. Of these, nine patients consented to take part and acted as subjects.

The study was approved by Showa University Ethics Committee and was carried out after all subjects received a full explanation of the aims and methods of the study and gave their consent to participate (approval no. 2012-020).

\section{Electromyograph attachment}

Masseter muscle activity was measured using an electromyograph (PowerLab; ADInstruments, Nagoya, Japan). Silver disk electrodes of $10 \mathrm{~mm}$ in diameter with bipolar leads (Duotrode; Morita Corp., Osaka, Japan) were adhered on both sides of the central part of the masseter muscle. The distance between electrodes was $21 \mathrm{~mm}$, and the electrodes were placed parallel to the direction in which the masseter muscle fibers run.

The activity of the masseter muscle, when subjects clenched their teeth at full strength with nothing interposed between the upper and lower teeth, was defined at $100 \%$ maximum voluntary contraction (MVC), and subjects were able to see the amount of muscle force displayed in numerical values through visual feedback.

\section{Measurement and analysis of subject dentition Measurement of occlusal loading and occlusal contact area using Occluzer}

Pressure-sensitive film (Dental Prescale 50H type R; Fuji Photo Film Co., Tokyo, Japan) for occlusal force diagnostic use was used to examine the occlusal contact area and occlusal load in the intercuspal position together. Masseter muscle activity (clenching strength) was set at 40, 60, 80, and $100 \% \mathrm{MVC}$, and subjects were measured three times at each of these clenching intensities using 
visual feedback. Subjects remained in a seated position, and the head on the headrest of the dental unit with the occlusal plane is parallel to the floor.

Subjects were instructed to open their mouths one finger width and the Prescale was inserted, and subjects were then instructed to slowly close their mouths and clench their teeth. The Prescale was interposed between the full dentition between the second molars on either side, and subjects were instructed to bite in the intercuspal position. Clenching on the Prescale was carried out for $3 \mathrm{~s}$, and considering the content of muscle fatigue, measurements were taken at 5 -min intervals.

The Prescale was kept in a cool, dark place for $24 \mathrm{~h}$; after which, the colored parts, their surface area, and their color density were analyzed using a dedicated analyzer (Occluzer FPD707 ${ }^{\circ}$; Fuji Photo Film Co., Tokyo, Japan). Occlusal force was analyzed by a software (DePROS-PC; GC, Tokyo, Japan), and these data were converted to pressure values. The occlusal loading and occlusal contact area for molars were then calculated.

\section{Measurement of occlusal contact area using BiteEye}

A material for checking accuracy of fit (Blue Silicone ${ }^{\oplus}$; GC, Tokyo, Japan) was used to examine the occlusal contact in the intercuspal position. The occlusal contact area was measured once at each clenching strength (20, 40, and $60 \%$ MVC) using visual feedback. Subject posture was the same as during Prescale measurement. Subjects were instructed to open their mouths one finger width and Blue Silicon was inserted above the lower row of teeth, and subjects were then instructed to keep it in the mouth for $15 \mathrm{~s}$. Subjects were instructed to keep the clenching strength of provisions while displaying the strength clenching on the monitor. Subjects clenched their teeth at the required strength for $30 \mathrm{~s}$ and kept the Blue Silicone in their mouths for a further $30 \mathrm{~s}$ until it hardened. Taking muscle fatigue into account, measurements were taken at 5-min intervals.

Thus, the Blue Silicone impressions obtained were examined using a BiteEye occlusal contact analyzer (BiteEye-I'; GC, Tokyo, Japan). The thickness of the Blue Silicone film at the points of occlusal contact was set at 10,20 , and $30 \mu \mathrm{m}$, and the occlusal contact area was calculated. When the thickness of the Blue Silicone film at the points of occlusal contact was set at $10 \mu \mathrm{m}$, Dental Prescale and Blue Silicone approximate in the occlusal contact area in the natural dentition [12].

While there have been studies in which the occlusal contact area was calculated from the silicone method, the thickness of silicone $(\mu \mathrm{m})$ that is defined as occlusion has not been quantitatively clarified. Okada et al. [16] found functional occlusal contacts can be recorded at the silicone thickness $30 \mu \mathrm{m}$. From the above sentence, the thickness of the Blue Silicone film at the points of occlusal contact was set at $10-30 \mu \mathrm{m}$.
Analysis of the results from Occluzer and BiteEye

The following calculations were performed:

1. Comparison of Occluzer and BiteEye in the occlusal contact area

2. Comparison of the occlusal contact area and occlusal load between the implant and same number of natural teeth on the contralateral side

3. Comparison of the occlusal contact area and occlusal load between molar areas of the implant side and contralateral side

4. Comparison of the occlusal contact area and occlusal load between molar areas of the implant side and contralateral side excluding the first molar

5. Comparison of the proportion of the occlusal contact area and occlusal load on the molar region accounted for by the prosthetic implant and contralateral tooth

Analysis of results from Occluzer and BiteEye was performed by two-way ANOVA with occlusal loading and occlusal area as dependent variables, and clenching strength and presence or absence of implant as betweensubject factors. The level of significance for multiple comparison tests was set at $5 \%$.

In addition, multiple comparison test was performed using the Bonferroni method. PASW Statistics 18 was used for all statistical calculations (SPSS, Tokyo, Japan).

\section{Results}

\section{Comparison of occlusal contact areas evaluated using Occluzer and BiteEye}

Occlusal contact area values obtained with BiteEye and Occluzer at clenching intensities from 20 to $100 \%$ MVC were compared (Fig. 1). At different clenching intensities, the contact area values of BiteEye with silicone thickness of $10 \mu \mathrm{m}(\mathrm{BE} 10 \mu \mathrm{m})$ were the most similar to the contact area values of Occluzer. Thus, comparisons

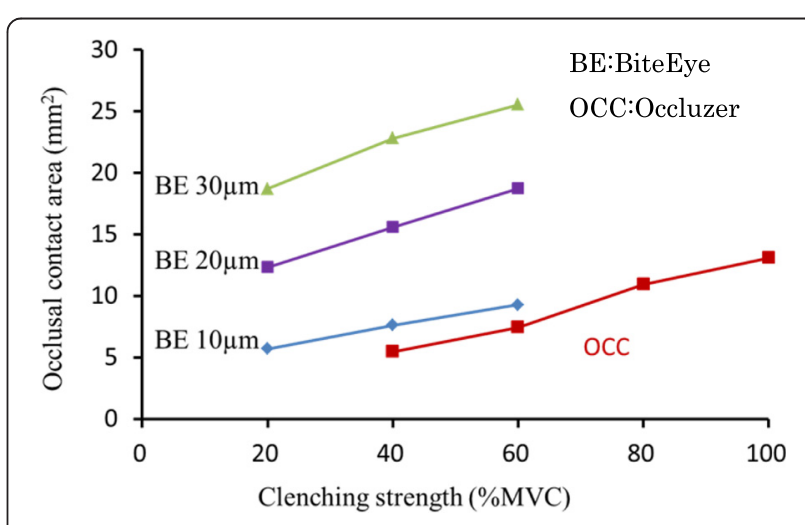

Fig. 1 Comparison of the occlusal contact area between Occluzer and BiteEye 
of occlusal contact area values between Occluzer and BiteEye were carried out with a silicone thickness of $10 \mu \mathrm{m}$.

\section{Comparison of implant region and contralateral tooth Comparison of occlusal contact area}

The occlusal contact area of implant prostheses increased with clenching strength and was always less than that of the contralateral tooth (Fig. 2). ANOVA results (Table 2) show a significant difference between implants and natural teeth with BiteEye $(P<0.05)$. Multiple comparison test showed that the occlusal contact area of implants was significantly smaller than the occlusal contact area of contralateral teeth with BiteEye at clenching intensities of 40 and $60 \% \mathrm{MVC}$ and Occluzer at clenching intensities of 80 and $100 \% \operatorname{MVC}(P<0.05)$.

\section{Comparison of occlusal load}

Occlusal load on implant prostheses increased with clenching strength and was always less than that on the contralateral teeth (Fig. 2). At $100 \% \mathrm{MVC}$, the occlusal load on the implant prosthesis was $47.7 \pm 39.0 \mathrm{~N}$ and that on the contralateral tooth was $81.4 \pm 41.1$ N. ANOVA results (Table 3 ) showed a significant interaction in the difference in occlusal load between implants and natural teeth, and clenching strength $(P<0.05)$. Multiple comparison test showed that the occlusal load was significantly smaller on the implant prostheses than on the contralateral teeth at clenching intensities of 80 and $100 \%$ MVC $(P<0.05)$.

The rate of increase in occlusal load from 40 to $100 \%$ MVC was $106.1 \%$ for the implant prosthesis and $127.4 \%$ for the contralateral tooth.

\section{Comparison of implant side and contralateral side molar regions}

Comparison of occlusal contact area in the molar region

The occlusal contact area in the molar region on the implant and contralateral sides increased with clenching strength and, at clenching intensity of $60 \% \mathrm{MVC}$ or above, was less on the implant side molar region (Fig. 3). ANOVA results (Table 4) showed no significant differences in occlusal contact area between the implant side molar region and the contralateral side molar region with BiteEye $(P=0.092)$. Multiple comparison tests showed no significant differences in the occlusal contact area between the molar region on the implant side and the molar region on the contralateral side at any clenching intensity with either BiteEye or Occluzer $(P>0.05)$.

\section{Comparison of occlusal load on the molar region}

Occlusal load on the implant side molar region and the contralateral side molar region increased with clenching strength and, at clenching intensity of $80 \% \mathrm{MVC}$ and above, was less on the implant side molar region (Fig. 3). At $100 \%$ MVC, the occlusal force on the implant side molar region was $212.7 \pm 57.6 \mathrm{~N}$ and on the contralateral side molar region was $274.4 \pm 111.5 \mathrm{~N}$. ANOVA results (Table 5) showed no significant differences in occlusal load between the implant side molar region and the contralateral side molar region $(P=0.278)$. Multiple comparison test showed no significant differences in occlusal load between the molar region on the implant side and the molar region on the contralateral side at any clenching intensity $(P>0.05)$.

\section{: Implant \\ : Same number of tooth on contralateral side}
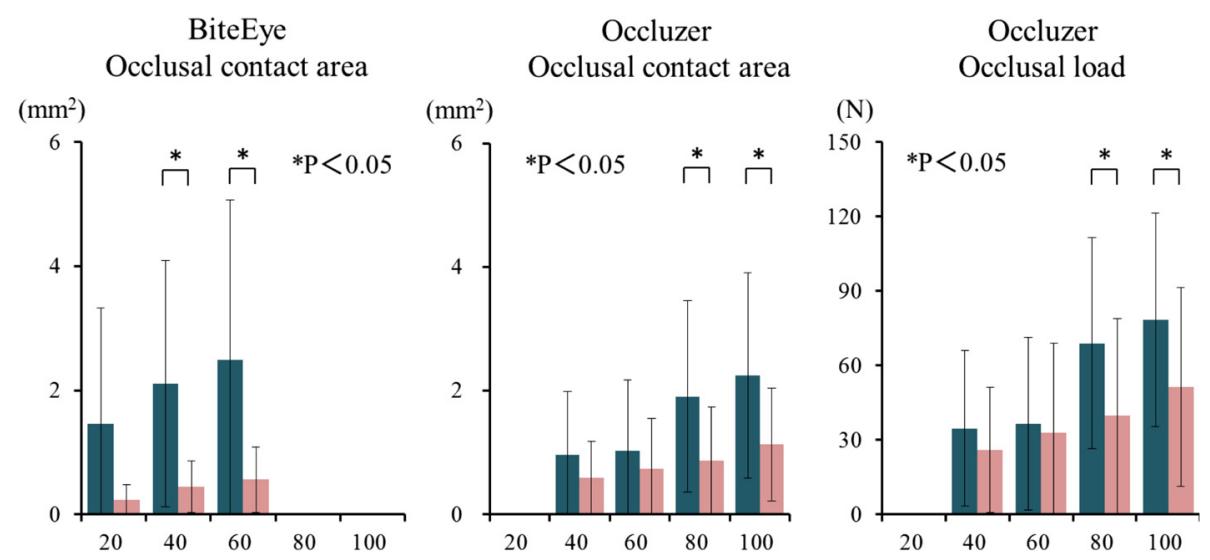

Clenching strength $(\% \mathrm{MVC})$

Fig. 2 Comparison of occlusal contact area and occlusal load between implant and contralateral tooth 
Table 2 Two-way ANOVA of the occlusal contact area of the implant prosthesis and contralateral tooth

\begin{tabular}{lllllll}
\hline & Source & $\begin{array}{l}\text { Sum of } \\
\text { square }\end{array}$ & $d f \begin{array}{l}\text { Mean } \\
\text { square }\end{array}$ & $F$ value & $P$ value \\
\hline Occluzer & $\begin{array}{l}\text { Implant or } \\
\text { natural tooth }\end{array}$ & 1941.060 & 1 & 1941.060 & 1.074 & 0.333 \\
& MVC & 424.098 & 3 & 141.336 & 1.958 & 0.147 \\
& Interaction & 262.581 & 3 & 87.527 & 1.345 & 0.283 \\
BiteEye & Implant or & 28.022 & 1 & 28.022 & 6.892 & 0.030 \\
& natural tooth & & & & & \\
& MVC & 3.374 & 2 & 1.687 & 1.927 & 0.178 \\
& Interaction & 0.916 & 2 & 0.458 & 0.827 & 0.455 \\
\hline
\end{tabular}

Comparison of implant side and contralateral side molar regions excluding the first molar Comparison of occlusal contact area in the molar region excluding the first molar

The occlusal contact area in the molar region excluding the first molar on the implant and contralateral sides increased with clenching strength (Fig. 4). ANOVA results (Table 6) showed no significant differences in the occlusal contact area between the implant side molar region and the contralateral side molar region with BiteEye $(P=0.400)$. Multiple comparison tests showed no significant differences in the occlusal contact area between the molar region excluding the first molar on the implant side and the molar region on the contralateral side at any clenching intensity with either BiteEye or Occluzer $(P>0.05)$.

\section{Comparison of occlusal load on the molar region excluding the first molar}

Occlusal load on the implant side molar region and the contralateral side molar region excluding the first molar increased with clenching strength (Fig. 4). At $100 \%$ MVC, the occlusal force on the implant side molar region was $165.0 \pm 45.8 \mathrm{~N}$ and on the contralateral side molar region was $193.3 \pm 85.8 \mathrm{~N}$. ANOVA results (Table 7) showed no significant differences in occlusal load between the implant side molar region and the contralateral side molar region $(P=0.990)$. Multiple comparison test showed no significant differences in occlusal load between the molar region excluding the first molar on the implant

Table 3 Two-way ANOVA of occlusal load of implant prosthesis and contralateral tooth

\begin{tabular}{lrrrrr}
\hline Source & $\begin{array}{l}\text { Sum of } \\
\text { square }\end{array}$ & $d f$ & $\begin{array}{l}\text { Mean } \\
\text { square }\end{array}$ & $F$ value & $P$ value \\
\hline Implant or natural tooth & 8732.252 & 1 & 8732.252 & 13.299 & 0.007 \\
MVC & $14,619.565$ & 3 & 4872.855 & 16.724 & 0.001 \\
Interaction & 2200.789 & 3 & 733.596 & 8.442 & 0.001 \\
\hline
\end{tabular}

side and the molar region on the contralateral side at any clenching intensity $(P>0.05)$.

\section{Comparison of proportion of occlusal contact area and occlusal load on the molar region accounted for by prosthetic implant and contralateral tooth}

With both BiteEye and Occluzer, the proportion of the occlusal contact area on the molar region overall accounted for by the prosthetic implant was less than the proportion accounted for by the contralateral natural tooth, and ANOVA showed this difference to be significant $(P<0.05)$ (Fig. 5, Table 8). Multiple comparison test also showed that the prosthetic implant accounted for a significantly smaller proportion of occlusal contact area at all clenching intensities other than 40 and $60 \%$ MVC with Occluzer $(P<0.05)$. With occlusal load as well, the prosthetic implant accounted for a significantly smaller proportion than the contralateral tooth, and at $100 \% \mathrm{MVC}$, the occlusal load on the prosthetic implant was $9.1 \%$ of the load on the molar region while the occlusal load on the contralateral tooth was $16.3 \%$. Multiple comparison test showed that the proportion of occlusal load on the molar region accounted for by the prosthetic implant was significantly smaller than that accounted for by the contralateral tooth at clenching intensities other than 40 and $60 \%(P<0.05)$.

No significant differences in the proportion of occlusal area or occlusal load on the molar region attributable to clenching strength were found with the prosthetic implant or the contralateral tooth with either BiteEye or Occluzer $(P>0.05)$ (Table 8$)$.

\section{Discussion}

\section{Subjects and dentition}

Males outnumbered females in the present study, but there have been no reports of differences in mean occlusal load between males and females in the 50-54 age group [17]. While there have been reports that in healthy, dentulous subjects, the total occlusal force in the molar region on one side is approximately $400 \mathrm{~N}$ at maximum clenching strength [18], in the present study, the mean occlusal force on the natural tooth side at $100 \%$ MVC was $274.4 \pm 111.5$ N. However, considering the subjects in the present study were of middle and old age, with mean age 49 years, the results probably have a certain validity [17-19].

Occlusal force is often measured across the whole jaw $[20,21]$. The Dental Prescale used for occlusal force measurement has a thickness of approximately $100 \mu \mathrm{m}$, and concern has been raised that this thickness might affect the results of occlusal force distribution measurement. However, as good reproducibility of results from the molar region has been reported, in the present study, the molar region from the first premolar to the second molar on both sides was examined $[11,12,22]$. 


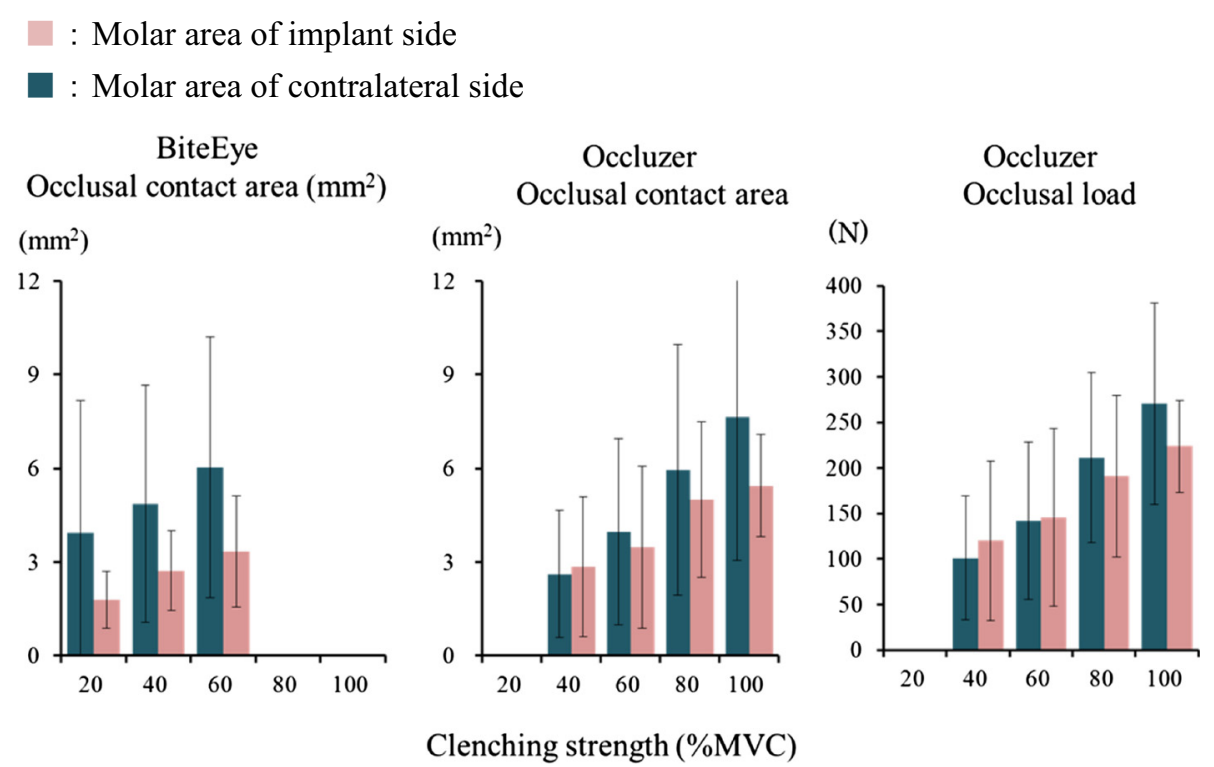

Fig. 3 Comparison of the occlusal contact area and occlusal load between the implant side molar region and contralateral side molar region

\section{Study methods}

\section{Examination of occlusal contact}

Materials such as wax, occlusal registration paper, and pressure-sensitive paper are used to examine occlusal contact. The present study utilized an examination method using silicone $[11,12,16]$. The Blue Silicone used in the present study is a cartridge type, which allows a nearly constant mixing ratio to be maintained, it has low viscosity and thus flows well, and it shows little dimensional change over time. It therefore fully met the requirements for a material for registration of occlusal contact status.

A prior study reported low reproducibility when using Dental Prescale at low clenching strength [22]. In addition, the use of Blue Silicone to take impressions at high clenching intensities of 80 and $100 \%$ MVC places a burden on subjects, as Blue Silicone takes a long time to harden. For these reasons, when measurements from low to high clenching strength were made in the present

Table 4 Two-way ANOVA of the occlusal contact area of the implant side molar region and contralateral side molar region

\begin{tabular}{llccccc}
\hline & Source & $\begin{array}{l}\text { Sum of } \\
\text { square }\end{array}$ & $d f$ & $\begin{array}{l}\text { Mean } \\
\text { square }\end{array}$ & $F$ value & $P$ value \\
\hline Occluzer & $\begin{array}{l}\text { Implant side and } \\
\text { contralateral side }\end{array}$ & 24.453 & 1 & 24.453 & 2.334 & 0.165 \\
& MVC & 159.749 & 3 & 53.253 & 26.918 & 0.000 \\
& Interaction & 16.135 & 3 & 5.378 & 3.786 & 0.024 \\
BiteEye & Implant side and & 51.431 & 1 & 51.431 & 3.674 & 0.092 \\
& contralateral side & & & & & \\
& MVC & 32.471 & 2 & 16.236 & 8.125 & 0.004 \\
& Interaction & 0.236 & 2 & 0.118 & 0.072 & 0.931 \\
\hline
\end{tabular}

study, Blue Silicone was used at clenching intensities of 20, 40, and $60 \% \mathrm{MVC}$, and Dental Prescale was used at 40, 60, 80, and $100 \%$ MVC.

\section{Measurement of occlusal load}

Occlusal load was measured with Dental Prescale and analyzed with Occluzer. The Dental Prescale system allows occlusal contact pressure to be measured quickly and easily across the dentition, and it is of enormous clinical utility because its accuracy and reproducibility in the molar region have been confirmed [16, 20, 22]. The technique using this sequence and also the method of cross-checking occlusal contact points using silicone and Dental Prescale are clinically useful and have been widely studied and reported [16, 20].

\section{Comparison of occlusal contact areas evaluated using Occluzer and BiteEye}

Blue Silicone with a thickness of $10 \mu \mathrm{m}$ and Dental Prescale gave similar values for the occlusal contact area at 40 and $60 \%$ MVC. These results probably have more validity than those reported by Imamura et al. [12].

Table 5 Two-way ANOVA of occlusal load of the implant side molar region and contralateral side molar region

\begin{tabular}{lcrrrr}
\hline Source & $\begin{array}{l}\text { Sum of } \\
\text { square }\end{array}$ & $d f$ & $\begin{array}{l}\text { Mean } \\
\text { square }\end{array}$ & $F$ value & $P$ value \\
\hline $\begin{array}{l}\text { Implant side and } \\
\text { contralateral side }\end{array}$ & 8578.099 & 1 & 8578.099 & 1.354 & 0.278 \\
MVC & $193,888.518$ & 3 & $64,629.506$ & 36.593 & 0.000 \\
Interaction & $30,575.358$ & 3 & 4016.245 & 3.153 & 0.430 \\
\hline
\end{tabular}


: Molar region of implant side excluding the first molar

: Molar region of contralateral side excluding the first molar

BiteEye

Occlusal contact area $\left(\mathrm{mm}^{2}\right)$ $\left(\mathrm{mm}^{2}\right)$

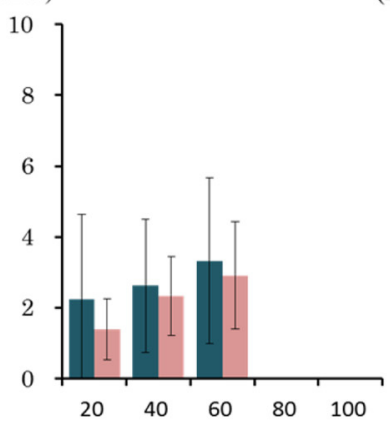

Occluzer

Occlusal contact area
Occluzer

Occlusal load
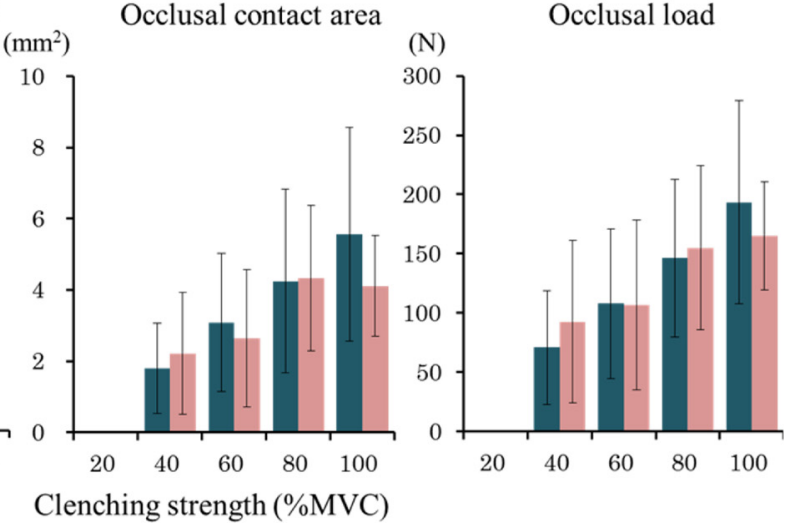

Fig. 4 Comparison of the first molar-eliminated occlusal contact area and load between the implant side molar region and contralateral side molar region

\section{Analysis of implant region Occlusal load on implant region}

An occlusal load of approximately $130 \mathrm{~N}$ on the first molar has been reported at maximum clenching strength in healthy, dentulous subjects [18]. In the present study, occlusal load on the mandibular first molar was somewhat less, at $81.2 \pm 41.1 \mathrm{~N}$. However, bearing in mind that the subjects in the prior study were in their $20 \mathrm{~s}$ whereas those in the present study were of middle to old age, with a mean age of 49 years, the present results are probably somewhat valid. Implant prostheses probably have greater occlusal load than natural teeth at higher clenching intensities because they lack the mechanical buffering function of the periodontal membrane. A study using two-dimensional finite element analysis on the mandibular first molar by Maezawa et al. [9] suggests that even if the occlusal surface of the prosthesis is made lower than the occlusal plane, the implant area may still

Table 6 Two-way ANOVA of the occlusal contact area of the implant side molar region and contralateral side molar region excluding the first molar

\begin{tabular}{llrrrrr}
\hline & Source & $\begin{array}{l}\text { Sum of } \\
\text { square }\end{array}$ & $d f$ & $\begin{array}{c}\text { Mean } \\
\text { square }\end{array}$ & $F$ value & $P$ value \\
\hline Occluzer & $\begin{array}{l}\text { Implant side and } \\
\text { contralateral side }\end{array}$ & 2.177 & 1 & 2.177 & 0.383 & 0.165 \\
& MVC & 90.753 & 3 & 30.251 & 25.800 & 0.553 \\
& Interaction & 9.011 & 3 & 3.004 & 2.209 & 0.113 \\
\multirow{5}{*}{ BiteEye } & Implant side and & 3.527 & 1 & 3.527 & 0.789 & 0.400 \\
& contralateral side & & & & & \\
& MVC & 15.013 & 2 & 7.541 & 10.636 & 0.001 \\
& Interaction & 0.790 & 2 & 0.395 & 0.661 & 0.530 \\
\hline
\end{tabular}

be subjected to excess occlusal load with increased clenching strength.

In the present study, however, the occlusal load on the implant prosthesis tended not to increase as much as the load on the contralateral tooth when the clenching strength was higher. A possible reason is that the dentists adjusted the implant prostheses with pressure displacement in mind, giving a smaller occlusal contact area so that there were fewer loading points than in the contralateral tooth $[4,21,22]$.

When considering the balance of occlusal load in the molar region, it is better to give the same occlusal load on both molar regions at $100 \% \operatorname{MVC}[12,16]$. However, in this study, the occlusal load was significantly smaller on the implant prosthesis than on the contralateral tooth at $100 \%$ MVC.

Measurement of occlusal contact area of implant prosthesis Dental Prescale has a thickness of approximately $100 \mu \mathrm{m}$, whereas Blue Silicone has less thickness and is therefore likely to give more accurate measurements of the occlusal contact area $[12,23]$. In addition, when the occlusal contact areas of implant prostheses and their contralateral

Table 7 Two-way ANOVA of occlusal load of the implant side molar region and contralateral side molar region excluding the first molar

\begin{tabular}{lrrrrr}
\hline Source & $\begin{array}{l}\text { Sum of } \\
\text { square }\end{array}$ & $d f \begin{array}{l}\text { Mean } \\
\text { square }\end{array}$ & $F$ value & $P$ value \\
\hline $\begin{array}{l}\text { Implant side and } \\
\text { contralateral side }\end{array}$ & 0.690 & 1 & 0.690 & 0.000 & 0.990 \\
MVC & $102,482.810$ & 3 & $34,160.937$ & 30.344 & 0.000 \\
Interaction & 6049.786 & 3 & 2016.595 & 1.336 & 0.286 \\
\hline
\end{tabular}


: Implant / All molars

: Same number of natural tooth on contralateral side / All molars
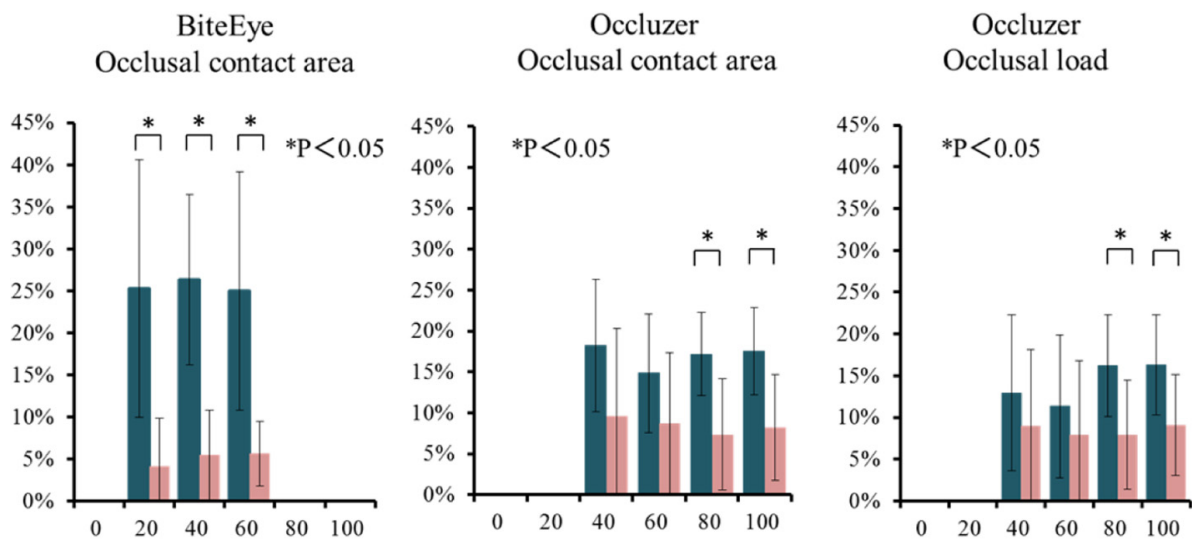

Clenching strength (\%MVC)

Fig. 5 Proportion of the occlusal contact area and occlusal load of the whole molar region accounted for by the implant prosthesis and by the contralateral tooth

teeth were compared, the occlusal contact area of the contralateral teeth was significantly greater at higher clenching strength. This is probably because occlusion between natural teeth results in greater displacement due to the presence of a periodontal membrane on both teeth. In addition, Koyama et al. [10] reported no significant differences between the molar region on the implant side and on the contralateral side, even when clenching strength varied. Similarly, when clenching strength varied in this

Table 8 Two-way ANOVA of the proportion of occlusal load and contact area for implants and first molars based on molar area

\begin{tabular}{clccccc}
\hline & Source & $\begin{array}{l}\text { Sum of } \\
\text { square }\end{array}$ & $d f$ & $\begin{array}{l}\text { Mean } \\
\text { square }\end{array}$ & $F$ value & $P$ value \\
\hline Occlusal load & & & & & & \\
Occluzer & $\begin{array}{l}\text { Implant or } \\
\text { natural tooth }\end{array}$ & 585.390 & 1 & 585.390 & 9.870 & 0.014 \\
& MVC & 53.901 & 3 & 53.901 & 1.768 & 0.220 \\
& Interaction & 46.872 & 3 & 46.872 & 3.791 & 0.870 \\
Occlusal & & & & & & \\
contact area & & & & & & \\
Occluzer & Implant or & 0.087 & 1 & 0.087 & 11.101 & 0.010 \\
& natural tooth & & & & & \\
& MVC & 0.003 & 3 & 0.003 & 1.202 & 0.305 \\
& Interaction & 0.004 & 3 & 0.004 & 2.178 & 0.178 \\
BiteEye & Implant or & 0.291 & 1 & 0.291 & 12.210 & 0.008 \\
& natural tooth & & & & & \\
& MVC & 0.040 & 2 & 0.040 & 1.287 & 0.290 \\
& Interaction & 0.000 & 2 & 0.000 & 0.070 & 0.798 \\
\hline
\end{tabular}

study, the occlusal contact area of the implant prosthesis did not increase than the contralateral tooth.

\section{Comparison of proportion of occlusal contact area and occlusal load in all teeth accounted for by prosthetic implant and contralateral tooth}

The proportion of occlusal force on the molar region of healthy dentition accounted for by the mandibular first molar on one side has previously been reported as $16 \%$ [20]. In the present study, however, the implant prosthesis accounted for a lower proportion of $9 \%$ of the occlusal force on the molar region. No significant differences in occlusal load were observed between sides, but the proportion of occlusal load on all teeth borne by the implant prosthesis was less than that borne by the contralateral tooth. However, there was no significant difference in occlusal load between both molar regions. It suggested that occlusal loading on both molar regions have been balanced.

\section{Future research}

The present study examined implant prostheses immediately after setting, but the occlusal contact of implant prostheses is believed to change over time as a result of factors such as extrusion of opposing teeth, abrasion from neighboring surfaces, and tooth attrition. In the future, we intend to use the results of the present study to carry out a prospective study that will survey the period of 1 year from immediately after setting, which is when problems are most common. This future study will have a greater number of subjects and will evaluate parameters such as mastication function. This study will aim to 
clarify how the occlusal contact of the implant prosthesis changes within the dentition and to draw up guidelines on this basis.

\section{Conclusions}

The results suggest that the occlusal contact of implant prostheses can be evaluated from low to high clenching intensities using Blue Silicone and Dental Prescale. There was a trend for implant prostheses to be adjusted such that immediately after setting the occlusal contact area, the occlusal load of the implant superstructure was less than that of the contralateral tooth. This is likely to be due to dentists taking into account the small tissue displaceability of implants.

The occlusal load on implant prostheses in a single intermediary mandibular first molar deficit tended to increase less with clenching strength than the load on the contralateral tooth. In addition, the proportion of the occlusal load on the whole dentition accounted for by the implant prosthesis was less than the proportion accounted for by the contralateral tooth. However, there was no significant difference in occlusal load between both molar regions. It suggested that occlusal loading on both molar regions has been balanced. The adequate occlusion on implant prosthesis has not been clear. However, we will be able to obtain clinical suggestions for the occlusion that should be given to prosthetic implants through a longitudinal follow-up study.

\section{Abbreviations}

MVC: maximum voluntary contraction; Prescale: Dental Prescale $50 \mathrm{H}$ type R; Occluzer: Occluzer FPD707; BiteEye: BiteEye-l; ANOVA: analysis of variance.

\section{Competing interests}

Yukihiko Okada, Yuji Sato, Noboru Kitagawa, Keiichiro Uchida, Tokiko Osawa, Yoshiki Imamura, and Mayumi Terazawa declare that they have no competing interests.

\section{Authors' contributions}

YO drafted the manuscript. YS contributed advice regarding the manuscript. $\mathrm{NK}, \mathrm{KU}, \mathrm{TO}, \mathrm{Yl}$, and MT checked and improved the manuscript. All authors read and approved the final manuscript.

\section{Acknowledgements}

The authors would like to express their deep appreciation to the staff of Implant Center at Showa University Dental Hospital for their help and cooperation. This study was supported by a Grant-in-Aid for Scientific Research from the Ministry of Education, Culture, Science, and Technology (Showa University Grant-in-Aid for Scientific Research (C)) (Grant Number 25463016). A partial summary of this thesis was presented at the 44th Annual Meeting of the Japanese Society of Oral Implantology in Tokyo, Japan in September 2014.

Received: 26 January 2015 Accepted: 25 May 2015

Published online: 04 July 2015

\section{References}

1. Hobo S, Hosoyama H. Occlusion for implant. Tokyo: Quintessence; 2006. p. 89-95 [in Japanese].

2. Schulte W. Implants and the periodontium. Int Dent J. 1995;45:16-26.

3. Miura H. Occlusal function and periodontal tissue. J Stomatol Soc Jpn. 2000;67:1-10 [in Japanese].

4. Morikawa O. Influence of occlusal contacts of implant on adjacent teeth and antagonists displacements. J Stomatol Soc Jpn. 2003;70:224-33 [in Japanese].
5. DARIO LJ. How occlusal forces change in implant patients: a clinical research report. J Am Dent Assoc. 1995;126:1130-3.

6. RANGERT BR, SULIIVAN RM, JEMET TM. Load factor control for implants in the posterior partially edentulous segment. Int J Oral Maxillofac Implnts. 1997;12:360-70.

7. Kim Y, Oh TJ, Misch CE, Wang HL. Occlusal considerations in implant therapy: clinical guidelines with biomechanical rationale. Clin Oral Implants Res. 2005;16:26-35.

8. Doi N, Inoue M, Inai T, Watanabe M, Sasaki K. Occlusal force on the dental arch in patients with dental implants at the free-end edentulous area. J Japanese Soc Oral Implantol. 2006;19:466-77.

9. Maezawa N, Wakabayashi N, Yokoyama S, Shiota M, Suzuki T. Influence of intensity of occlusal contact in implant-retained single restoration on stress distributions of crown surface and supporting bone. J Japan Prosthodontic Soc. 2007;51:582-91 [in Japanese].

10. Koyama K, Kusumoto T, Kawazoe T. Occlusal contacts in intercuspal position of prostheses without stress absorbing elements on osseointegrated implants. J Japanese Soc Oral Implantol. 2005;18:563-71.

11. Okuyama T, Sato Y, Ozawa K, Kitagawa N, Uchida K. Occlusion of prostheses supported by an implant for a single intermediate missing posterior tooth with satisfactory prognosis. J Japanese Soc Oral Implantol. 2010;23:209-19 [in Japanese].

12. Imamura $Y$, Sato $Y$, Kitagawa N, Uchida K, Osawa T, Omori M, Okada Y. Influence of occlusal loading force on occlusal contacts in natural dentition. J Prosthodont Res. 2015:59:113-120.

13. Kohno S, Shiva H, Nakano M, Furuya R, Mayanagi A, Minagi S, Kobayashi H. A guideline of three dentistry area disorders. J Japan Prosthodontic Soc. 2002;46:577-627 [in Japanese].

14. Smith DE, Zarb GA. Criteria for success of osseointegrated endosseous implant. J Prosthet Dent. 1989;62:567-72.

15. Albrektsson R, Zarb GA. Current interpretations of the osseointegrated response: clinical significance. Int J Prosthodont. 1993;6:95-105.

16. Daizo O. Teeth displacements and occlusal contacts depending on clenching force. J Jpn Prosthodont Soc. 1998;42:1013-23.

17. Kurosawa M. Studies on human biting force gender differences and lateral dominance with development and growth. J Japanese Academy Occlusion Health. 2002;8:8-16 [in Japanese].

18. Tanaka M, Johnston EL. The prediction of the size of unerupted canines and premolars in a contemporary orthodontic population. J Am Dent Assoc. 1974;88:798-801.

19. Hidaka O, Iwasaki M, Saito M. Influence of clenching intensity on bite pressure. J Dent Res. 1999;78:1336-44

20. Hattori $Y$, Satoh C, Watanabe M. Bite force distribution on dental arch during clenching. J Japanese Soc Stomatognathic Function. 1996;2:111-17 [in Japanese].

21. Suzuki T, Kumagai H, Yoshitomi N. Clinical evaluation of measuring system of occlusal force. J Stomatol Soc Jpn. 1994;61:437-45 [in Japanese].

22. Yamaguchi $T$, Hisatsune $Y$, Kimura T. Examination of occlusal contacts by using "Dental Prescale" special reference to the rate of detected occlusal contacts in the intercuspal position. J Prosthodont Res. 1995;39:1113-20 [in Japanese].

23. Baba K, Tsukiyama Y, Clark GT. Reliability, validity, and utility of various occlusal measurement methods and techniques. J Prosthet Dent. 2000;83:83-9.

\section{Submit your manuscript to a SpringerOpen ${ }^{\odot}$ journal and benefit from:}

- Convenient online submission

- Rigorous peer review

- Immediate publication on acceptance

- Open access: articles freely available online

- High visibility within the field

- Retaining the copyright to your article

Submit your next manuscript at $>$ springeropen.com 